\title{
HIV testing among men who have sex with men: how effective can frequent testing be?
}

\author{
Authors: Maarten Reitsema',2, Maartje Visser', Birgit van Benthem', \\ Janneke Heijne', Maria Xiridou' \\ 1. National Institute for Public Health and the Environment, the Netherlands. \\ 2. Department of Medical Statistics and Bioinformatics, Leiden University Medical \\ Center, The Netherlands. \\ Contact: maria.xiridou@rivm.nl
}

\section{Background}

The benefits of antiretroviral therapy for HIV infection can be enhanced if HIV-infected individuals are diagnosed early after infection. To limit late diagnoses and to shorten the time between infection and diagnosis, efforts are made internationally to improve HIV testing.

\section{Objective}

To assess the impact of frequent HIV testing on HIV transmission among men who have sex with men (MSM) in the Netherlands.

\section{Methods}

- We developed a transmission model that describes the transmission of HIV and N. gonorrhoeae via condomless anal sex among MSM.

- The model was calibrated to data on HIV diagnoses and anogenital gonorrhoea positivity rate in the Netherlands in 2007-2014.

- We compared four testing scenarios:

1. $20 \%$ of MSM tested six-monthly (current testing rates);

2. $50 \%$ of all MSM tested six-monthly;

3. $50 \%$ of MSM with at least 10 partners in the preceding 6 months tested six-monthly;

4. $50 \%$ of MSM diagnosed with gonorrhoea in the preceding 12 months tested six-monthly.

\section{Results}

- By increasing HIV-testing, the number of HIV diagnoses can be very high in the first year and decline thereafter. The number of diagnoses declines from 830 in 2018 to 360 diagnoses in 2027 , with $50 \%$ six-monthly testing among all MSM (Figure 1a).

- Many more HIV tests were carried out with $50 \%$ six-monthly testing among all MSM, compared to the number of tests with the other testing rates (Figure $1 \mathrm{~b}$ ).

- The number of HIV tests needed per new HIV diagnosis in 2027 was the highest with $50 \%$ six-monthly testing among all MSM (532 tests/diagnosis) and lowest with 50\% testing among MSM who had gonorrhoea (230 tests/diagnosis) (Figure 1c).

- After ten years, the percentage of HIV-infected MSM being undiagnosed can be slightly reduced to $3.5 \%$ with $50 \%$ six-monthly testing among all MSM, compared to $6.4 \%$ with the current testing rates (Figure 2 ).

\section{Conclusions}

Six-monthly screening can be effective in identifying high numbers of HIV-infected MSM, especially in the beginning. In the long run, targeting screening at high-risk MSM can be more efficient in terms of numbers of tests needed to diagnose one HIV infection.

\section{Published by}

National Institute for Public Health

and the Environment

P.O. Box 1 | 3720 BA Bilthoven

The Netherlands

www.rivm.nl/en

\section{Acknowledgements}

This study was supported by a grant

from Aidsfonds (project 2014037)
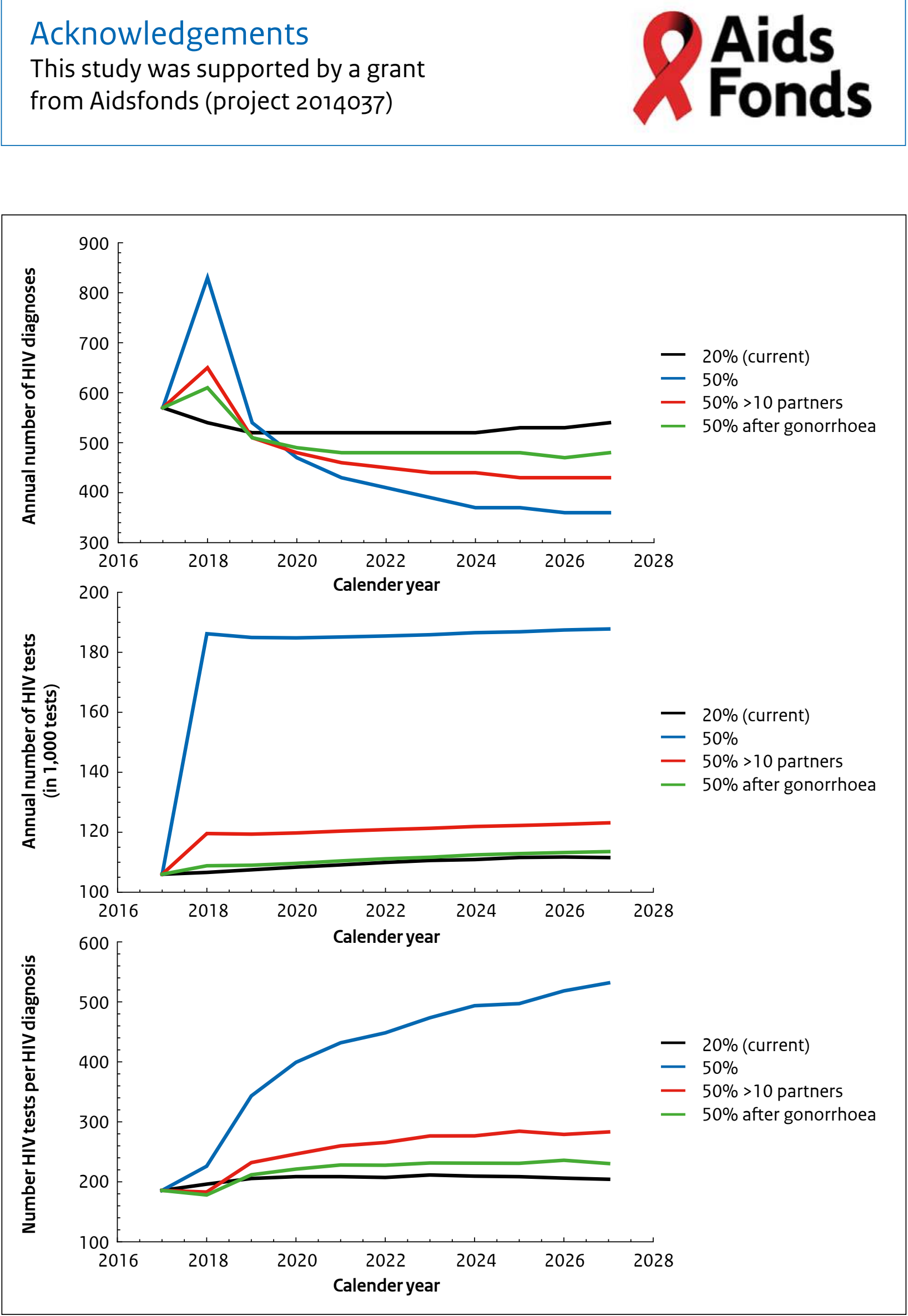

Figure 1. (a) The annual number of HIV diagnoses; (b) the number of HIV tests carried out each year; (c) the number of HIV tests carried out during each year per individual diagnosed with HIV during that year. Black: $20 \%$ of MSM tested six-monthly (current testing rates); blue: $50 \%$ of all MSM tested six-monthly; red: $50 \%$ of MSM with > 10 partners in the preceding 6 months tested six-monthly; green: $50 \%$ of MSM diagnosed with gonorrhoea in the preceding 12 months tested six-monthly.

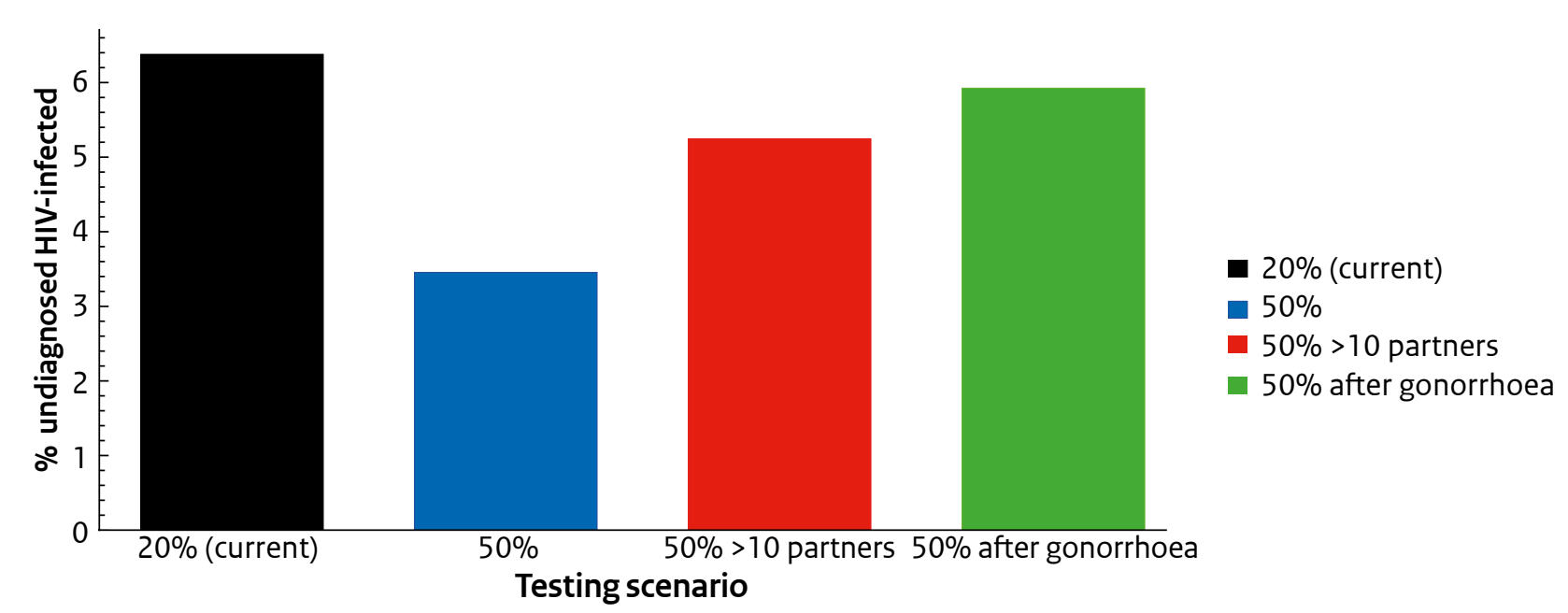

Figure 2. The percentage of HIV-infected MSM that is undiagnosed in 2027. Black: $20 \%$ of MSM tested six-monthly (current testing rates); blue: $50 \%$ of all MSM tested six-monthly; red: $50 \%$ of MSM with >10 partners in the preceding 6 months tested six-monthly; green: $50 \%$ of MSM diagnosed with gonorrhoea in the preceding 12 months tested six-monthly. 